\title{
Requerimientos hídricos de la zanahoria (D. carota L.) durante tres etapas de su desarrollo
}

\section{Water requirements of carrot (D. carota L.) in three stages of development}

Fecha de recepción:7 de marzo de 2015

Fecha de aprobación: 15 de junio de 2015
Fabio Emilio Forero-Ulloa ${ }^{1}$

Germán Eduardo Cely-Reyes ${ }^{2}$

Edilson Emilio Neira-Rodríguez ${ }^{3}$

\section{Resumen}

La zanahoria (D. carota L.) es uno de los principales productos del departamento de Boyacá, participa con 1093 ha, especialmente en los municipios de Ventaquemada, Samacá y Tunja. Los híbridos Chantenay tienen en Ventaquemada un rendimiento promedio de 20 t.ha ${ }^{-1}$, que es bajo comparado con el de la Sabana de Bogotá, de 28.1t.ha ; las limitantes en la producción de la zona son: falta de riego, semillas de altas exigencias agronómicas (adecuada mecanización del suelo, fertilización apropiada...) y presencia de patógenos y arvenses. Con el objetivo de buscar los requerimientos hídricos del cultivo de zanahoria en tres fases fenológicas (crecimiento, elongación y madurez del órgano cosechable), el estudio se desarrolló en el segundo semestre de 2014, en Ventaquemada, vereda Bojirque; la evaluación de las fases se realizó bajo un diseño de muestreo estratificado con muestreos destructivos efectuados cada 30 días a partir de la siembra. El valor del coeficiente de cultivo $(\mathrm{Kc})$ se determinó a través de la metodología propuesta por la FAO. Se instalaron dos lisímetros de $1 \mathrm{~m}^{3}$ de capacidad, con el fin de establecer el consumo hídrico del cultivo. La evapotranspiración del cultivo de referencia (ETo) fue obtenida con los datos climáticos tomados por la estación meteorológica inalámbrica Ambient Tiempo WS-2080 instalada en el sitio, los cuales se analizaron a través de la ecuación de Penman-Monteith, con el apoyo del software Cropwat. El Kc para cada etapa fenológica fue: fase crecimiento, 0.14; fase elongación, 0.29, y fase madurez órgano cosechable, 0.55 .

Palabras clave: Hortaliza, coeficiente de cultivo, evapotranspiración de cultivo, riego, producción de zanahoria.

1 M.Sc. Universidad Pedagógica y Tecnológica de Colombia (Tunja-Boyacá, Colombia). fabio.forero@uptc.edu.co.

2 M.Sc. Universidad Pedagógica y Tecnológica de Colombia (Tunja-Boyacá, Colombia).

3 M.Sc. Profesional independiente (Tunja-Boyacá, Colombia). 


\begin{abstract}
Among the main production systems in the department of Boyacá is the carrot $(D$. carota L.), which participates with 1093 ha, especially in Ventaquemada, Samacá and Tunja municipalities. The Chantenay hybrids Ventaquemada have an average yield of $20 \mathrm{t}^{-\mathrm{ha}^{-1}}$, which is low compared to the Sabana de Bo-

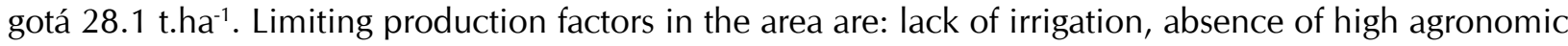
requirements seeds (appropriate mechanization soil, proper fertilization...), and presence of pathogens and weeds. The purpose of this paper was to find the water requirements of the crop in three phenological phases (growth, maturity and Elongation harvestable organ). It was developed in the second half of 2014, in Ventaquemada, Bojirque path; the assessment phase was carried out under a stratified sampling design, destructive samples were taken every 30 days from planting. The Kc value was determined using the methodology proposed by FAO.

Two lysimeters of $1 \mathrm{~m}^{3}$ capacity were installed in order to establish the water consumption of the crop. ETo was obtained with the climatic data taken by the wireless weather station Ambient Weather WS-2080 installed on the site, which is analyzed through the Penman-Monteith CROPWAT leaning the software. The Kc for each phenological stage was: 0.14 growth phase, 0.29 elongation phase, and 0.55 maturity phase of harvestable organ.
\end{abstract}

Keywords: Vegetable, crop coefficient, evapotranspiration crop, irrigation, carrot production. 


\section{Introducción}

La zanahoria (D. carota L.) es una planta bienal, herbácea, de la familia Apiáceae; la que se conoce hoy fue desarrollada por los holandeses en el siglo XVII. Los mayores productores en el 2013 fueron China (39.7\% del total mundial), Rusia, Ucrania e India, los cuales producen, en conjunto, un poco más del 50\% del total mundial (3). En Colombia, en el 2013, el área sembrada fue de 8347 ha, y en Boyacá, para este mismo año, se cultivaron 1093 ha, 540 de ellas en Ventaquemada (4).

La planta presenta crecimiento vegetativo y reproductivo, la radícula origina una raíz pivotante, su engrosamiento inicia en la parte superior de la raíz hacia la punta, alcanzando su máximo peso a los 120 días después de su emergencia. La fase reproductiva comprende la formación de flores, frutos y semillas. En la fase I, el crecimiento es lento en sus primeros estadios de desarrollo vegetativo, va desde la germinación hasta llegar a obtener tres hojas verdaderas y un diámetro superior de la raíz de 0.5 $\mathrm{mm}$. En la fase II Ilega a obtener 8 hojas verdaderas y un diámetro superior de la raíz del $60 \%$ del que se alcanza al final. La fase III finaliza con 10 hojas, un diámetro superior de la raíz promedio de 5.9 $\mathrm{cm}$ y un peso promedio de $250 \mathrm{~g}$ (2). La raíz de la zanahoria se diferencia según dimensión (larga, media, corta), forma (cónica, cilíndrica, fusiforme), tipo de ápice (redondeado, filififorme, obtuso), color externo (rojo, anaranjado, amarillo, violáceo) y consistencia (fibrosa, leñosa, crocante) (1).

Determinar el requerimiento de agua en cada etapa fenológica de la zanahoria es fundamental para alcanzar un mayor rendimiento del cultivo (5). La cantidad y frecuencia de riego se ven influidas por la etapa de desarrollo del cultivo y el medioambiente.

En ensayos en Mendoza (Argentina), usando riego por goteo, se ha llegado a producciones de 85 t.ha1 , totalizando $1080 \mathrm{~mm}$ de riego (6).

Los efectos combinados de transpiración del cultivo y evaporación del suelo se integran en el coeficiente único del cultivo (Kc). Así, el Kc incorpora las características del cultivo y los efectos prome- dios de la evaporación en el suelo, constituyendo una excelente herramienta para la planificación y programación de calendarios de riego en periodos mayores a un día. El objetivo del presente trabajo consistió en buscar los requerimientos hídricos del cultivo de zanahoria en tres fases fenológicas en el municipio de Ventaquemada.

\section{Materiales y métodos}

\section{Localización geográfica del estudio}

El estudio se desarrolló en Ventaquemada, departamento de Boyacá, vereda Bojirque, ubicada a $5^{\circ}$ $23^{\prime} 35.69^{\prime \prime}$ latitud Norte y $73^{\circ} 27^{\prime} 36.41^{\prime \prime}$ longitud Oeste, a una altitud de 2910 msnm, entre los meses de junio y diciembre de 2014; las condiciones climáticas de la zona en este periodo (5 meses) fueron: precipitación, $367.7 \mathrm{~mm}$; temperatura máxima, $16.8^{\circ} \mathrm{C}$, y temperatura mínima, $7.5^{\circ} \mathrm{C}$ (promedio, $10.9^{\circ} \mathrm{C}$ ), y humedad relativa, $87.3 \%$ en promedio.

\section{Toma de muestras}

El material vegetal utilizado fue la especie $D$. carota, híbrido Córdoba, de raíces cónicas, tipo Chantenay, longitud raíz $14-16 \mathrm{~cm}$; se adapta entre los 1800 y los2600 msnm; días a cosecha,120-125 días en fresco. El experimento se estableció en un Typic Melanudans con $\mathrm{pH}$ de $4.69,14.89 \%$ de materia orgánica, 6.35 ppm de fósforo Bray II y 5.98, $1.25,1.69$ y $1.1 \mathrm{cmol}_{\mathrm{C}} \cdot \mathrm{kg}^{-1}$ de calcio, magnesio, potasio y aluminio, respectivamente. Al momento de la preparación del suelo se aplicó enmienda de cal dolomita en dosis de 1.5 t.ha ${ }^{-1}$, y fertilización fraccionada, en presiembra y a los 30 días después de la germinación, para obtener un rendimiento de 28 t.ha $^{-1}$. Distancia de siembra entre surcos, 0.20 $\mathrm{m}$, y entre sitios, $0.15 \mathrm{~m}$, depositando 1 semilla por sitio, para un total de $1.25 \mathrm{~kg}$ de semilla por ha. El manejo fitosanitario se realizó de acuerdo con la evaluación de incidencia y severidad (enfermedades), umbral de daño de económico (plagas) y las arvenses en pre y posgerminación de la zanahoria. El riego se realizó teniendo en cuenta la evapotranspiración del cultivo de referencia (ETo) con la evapotranspiración del cultivo (ETc). La evaluación fenológica se inició con la germinación, siguiendo 
el desarrollo del cultivo a través de observaciones in vivo cada 7 días, llevando un registro escrito y fotográfico semanal, de acuerdo con la metodología propuesta por Bleiholder et al. (7). Se tomaron aleatoriamente 5 plantas para medir cada una de las variables propuestas. Las variables analizadas y cuantificadas fueron: altura de la planta $(\mathrm{cm})$, número de hojas (unidades), diámetro de la raíz $(\mathrm{cm})$, longitud de la raíz (cm) y peso fresco y seco de la raíz (g). Los datos obtenidos se compararon con la escala general de fenologías existente para todas las especies vegetales y la propuesta por Bleiholder et al. (7), donde muestra la escala específica de desarrollo de las hortalizas cuyo órgano cosechable es la raíz.

\section{Recolección de datos}

Para estimar el Kc en cada fase se siguió la metodología propuesta por la FAO (8), utilizando los datos que diariamente registraba la estación climatológica y los tomados en campo.

Para determinar la evapotranspiración del cultivo (ETc) se instalaron en el lote de experimentación dos lisímetros, fabricados en fibra de vidrio, con capacidad de $1 \mathrm{~m}^{3}$ y con $25 \mathrm{~cm}$ de fondo; con una lámina con orificios, por los que ingresaba al lisímetro el agua de drenaje que no fue utilizada por el cultivo; esta lámina se cubrió de grava, y luego se llenó el lisímetro con el mismo suelo extraído, teniendo precaución de no invertir las capas del suelo, para así mantener el perfil original de este; se niveló, y se dejó un borde de $5 \mathrm{~cm}$ sobre el nivel del suelo para evitar la entrada de agua de escorrentía. Una vez puesto el suelo dentro de lisímetro, se realizó la siembra. El agua que drenó al fondo del lisímetro se evacuó por medio de un tubo de PVC instalado en una esquina de este, con una bomba de succión. Para determinar la ETc (método del lisímetro) se tuvieron en cuenta los siguientes parámetros: precipitación diaria, lámina de riego, drenaje que se extrajo del lisímetro y los cambios en el contenido de humedad del suelo. Se hizo un monitoreo para cuantificar las variables contempladas en la ecuación del balance hídrico, de la que se despejo ETc (9).
Ecuación del balance hídrico: $\mathbf{P}+\mathbf{I}+/-\mathbf{R o}=\mathbf{E T c}$ $+/-\mathrm{D}+/-\Delta \mathrm{W}$

\section{Despejando: $\mathbf{E T C}=\mathbf{P}+\mathbf{I}+\mathbf{D}+/-\Delta \mathbf{W}+/-\mathbf{R o}$}

Dónde: $\mathbf{P}=$ Precipitación media a través de los pluviómetros, en milímetros.

I = Lámina de riego aplicada, en riegos. Se aplicó la cantidad de agua evaporada por día.

ET = Evapotranspiración. Dato obtenido de la estación climatológica.

D = Percolación o agua de drenaje en milímetros. Extraída del fondo de los lisímetros con la bomba de succión, generalmente después de una Iluvia fuerte.

$\Delta \mathbf{W}=$ Cambios en el contenido de humedad del suelo. Se tomó diariamente mediante las sondas de humedad.

$\mathbf{R o}=$ Escorrentía. Se controló con las paredes del lisímetro, entonces se tomó como cero).

La ETo se calculó a través del programa Cropwat (9), con los datos climáticos suministrados por la estación meteorológica. Se emplearon datos de temperatura, humedad, velocidad del viento y radiación solar para estimar la ETo, aplicando la ecuación de Penman-Monteith.

\section{Resultados y discusión}

La $D$. carota presenta los mayores rendimientos en temperaturas entre $13^{\circ}$ y $18^{\circ} \mathrm{C}$; la forma típica de la $D$. carota se produce a una temperatura de 18 ${ }^{\circ} \mathrm{C}$; a $13{ }^{\circ} \mathrm{C}$ es más larga y delgada, y a $24{ }^{\circ} \mathrm{C}$ es más corta y gruesa; el color amarillo de las raíces aparece al mes de la siembra, llegándose a la producción máxima de caroteno a los tres meses; el contenido de azúcar aumenta a los tres meses de siembra y se mantiene constante hasta la cosecha (10).

Fase I. Crecimiento: La fase duró 31 días, desde la germinación hasta formar 2 hojas verdaderas; tiempo que corresponde al $24.8 \%$ del total del ci- 
clo. La zanahoria presentó un crecimiento lento en sus primeros estadios de desarrollo vegetativo, lo cual concuerda con lo reportado por Suojala (1); se caracterizó por la elongación rápida de la raíz principal, y por una acumulación mínima de masa en fresco (1.8 g); no hubo cambios fenotípicos significativos; los mayores cambios se observaron en la longitud de las plantas; las hojas alcanzaron un valor de $6.4 \mathrm{~cm}$, y la raíz, un valor de $9.2 \mathrm{~cm}$; el diámetro ecuatorial del hombro alcanzó $0.5 \mathrm{~cm}$ (Fig. 1).

Fase II. Elongación: La fase duró 58 días, desde el día 31 después de la germinación hasta el día 89 dde, los cuales corresponden al $46.4 \%$ del tiempo total del ciclo. Durante la fase se observó un aumento importante en la mayoría de las variables fisiológicas estudiadas, principalmente en el diámetro del hombro de la raíz, llegando a un $60.6 \%$ del diámetro final; el número total de hojas Ilegó a $80 \%$ del total de hojas a cosecha; la acumulación de masa en fresco total confirma lo descrito por Vega et al. (11) en la provincia de Cartago, Costa Rica, en cuanto a que en el lapso de 61 a 97 dde hay un aumento constante en el diámetro de la raíz y en el número de hojas. Los mayores cambios se observaron en la longitud de la planta; las hojas y la raíz alcanzaron un valor de 33.5 y $18.8 \mathrm{~cm}$, respectivamente; el número de hojas alcanzó un valor máximo de 8 hojas, y el diámetro ecuatorial del hombro alcanzó $3.58 \mathrm{~cm}$ (Fig. 1), Esto concuerda con Strandberg (12), quien encontró en suelos orgánicos de la Florida que el crecimiento foliar y radicular durante los primeros 90 días la curva de crecimiento fue lineal.

Fase III. Madurez del órgano cosechable: La fase, final del ciclo, inició a los 89 dde y terminó a los 125 dde, es decir, duró 36 días, correspondientes al $28.8 \%$ del total del ciclo. Durante esta, los mayores cambios se observaron en el diámetro de la raíz y en el peso, que presentaron un aumento marcado (Fig. 1D); la raíz alcanzó un diámetro ecuatorial máximo de $5.9 \mathrm{~cm}$, y un peso máximo de 250 g, evento que ocurrió a los 125 dde, similar a lo reportado en Filandia (1); el crecimiento de las hojas se estabilizó, al igual que el número final de hojas, que alcanzó un valor máximo de 10; la longitud de la raíz finalizó con un valor de $19.74 \mathrm{~cm}$, superior al promedio de 10 a $15 \mathrm{~cm}$ reportado por Pinzón (10), y el diámetro ecuatorial del hombro alcanzó $5.9 \mathrm{~cm}$ (Fig. 1). Estos resultados concuerdan con Matute y Hoyos (13), para quienes el máximo potencial para el diámetro de la raíz $(4 \mathrm{~cm})$ y el peso $(150 \mathrm{~g})$ se dan, aproximadamente, durante el último cuarto del crecimiento de la cosecha en la provincia de Segovia, España, y divergen de lo citado por Enciso (14), para quien el diámetro ecuatorial del hombro máximo a cosecha fue de $3,4 \mathrm{~cm}$ y la longitud máxima de la raíz fue $13.78 \mathrm{~cm}$. El número de hojas a cosecha coincide con Nozumu (15), quien menciona que la zanahoria presenta entre 6 y 15 hojas por planta. 

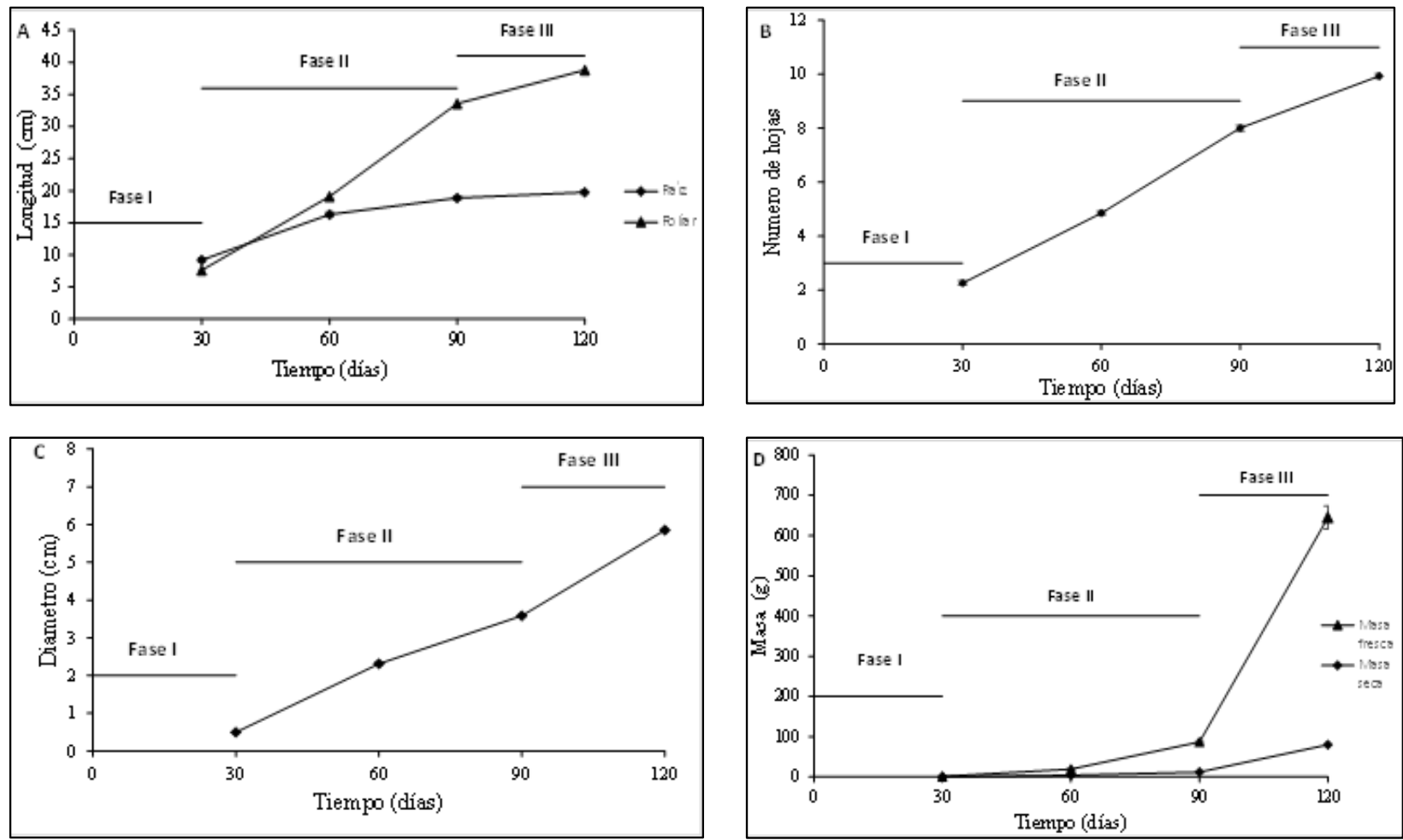

Figura 1. Principales variables morfométricas y fisiológicas analizadas para determinar las fases fisiológicas del cultivo de zanahoria. A. Longitud de la raíz y parte foliar. B. Número de hojas. C. Diámetro ecuatorial del cuello. D. Masa total en fresco y en seco.

El valor de Kc varió en el ciclo (Fig. 2A). En la fase I, el Kc fue, en promedio, de 0.14; en la fase II se reportó un Kc promedio de 0.29 , y en la fase III el Kc fue de 0.55. Los valores de Kc obtenidos en el presente estudio están en el rango de 0.09 a 0.82 (Fig. 2A), y difieren de los reportados por Allen et al. (16) en plantas de $30 \mathrm{~cm}$ de altura, los cuales son: $0.7,1.05$ y 0.95 , para las etapas inicial, intermedia y final de desarrollo, respectivamente; además, son inferiores a los citados por Fernández et al. (17) en 4 fases de desarrollo (inicial, desarrollo, media y maduración),en Andalucía, España: 0.45, $0.75,1.05$ y 0.9 , respectivamente.

Las etapas del cultivo no son iguales en todas las latitudes, en consecuencia, los Kc y escalas de fenología que sugiere la literatura son útiles como una guía general y para propósitos comparativos, por lo que debe generarse información local (18).
Generalmente, la zanahoria se cultiva bajo riego, dado que la combinación de temperaturas y humedad elevadas reduce significativamente el rendimiento, y requiere en su ciclo de 600 a $1700 \mathrm{~mm}$ (19); para el caso de estudio, los requerimientos hídricos de esta hortaliza fueron $106.78 \mathrm{~mm}$ durante la totalidad del ciclo, esto se debe a que la zona donde se desarrolló la investigación corresponde, según Holdridge, a Bosque húmedo Montano (bhM) y está a 2910 msnm.

Bajo las condiciones climáticas existentes durante el ciclo de cultivo, la ETo presentó una variabilidad diaria que va de 2.08 a $3.14 \mathrm{~mm}$. día ${ }^{-1}$, influyendo directamente en los valores de ETc y Kc. Esta variabilidad se debió, principalmente, a la fluctuación de la baja radiación solar, la cual se presentó en la mayor parte del ciclo de cultivo. En la fase I, la ETo presentó un valor promedio de $2.72 \mathrm{~mm}$ día $^{-1}$, y la ETc, al inicio de la fase, presentó valores diarios medios de $2.7 \mathrm{~mm} \mathrm{día}^{-1}$, pero al final llegó 
a $2.61 \mathrm{~mm} \mathrm{día-1}$ (Fig. 2); de acuerdo con lo anterior, y teniendo en cuenta que $\mathrm{Kc}$ es el resultado de la relación entre las dos evapotranspiraciones, en la primera fase del cultivo se presentaron valores bajos, que alcanzaron su punto máximo en 0.71. En la fase II, la ETc se estabilizó con valores promedio de $0.8 \mathrm{~mm} \mathrm{día}^{-1}$, en pleno crecimiento, presentando la menor variación de todo el ciclo, el Kc presentó valores de 0.16 , que aumentó gradualmente y llegó a 0.42 al final de la fase, con un promedio de 0.29. La fase III culmina con la cosecha; el cultivo sufre un proceso de pausa, y a partir de

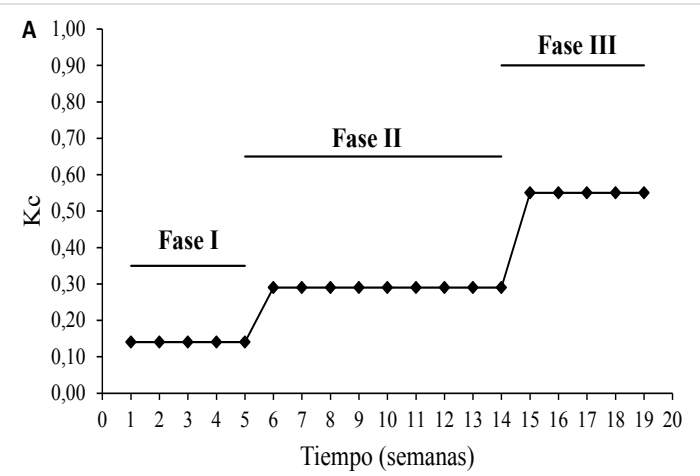

este momento tanto la ETc como el Kc se reducen y van perdiendo su capacidad fisiológica. La ETo reportó su punto más alto a los 112 dde, Ilegando a $2.35 \mathrm{~mm} \mathrm{día}^{-1}$. Al inicio de la fase III se evidenció que las plantas alcanzaron el máximo Kc 0.82, lo que coincide con el mayor desarrollo de área foliar, y al finalizar obtuvo un valor de Kc 0.27. La ETc muestra valores promedio de $1.6 \mathrm{~mm} \mathrm{día}^{-1}, \mathrm{y}$ la ETo evidencia un comportamiento en descenso durante la fase, reportando un valor promedio de $2.8 \mathrm{~mm} \mathrm{día}^{-1}$ (Fig. 2).

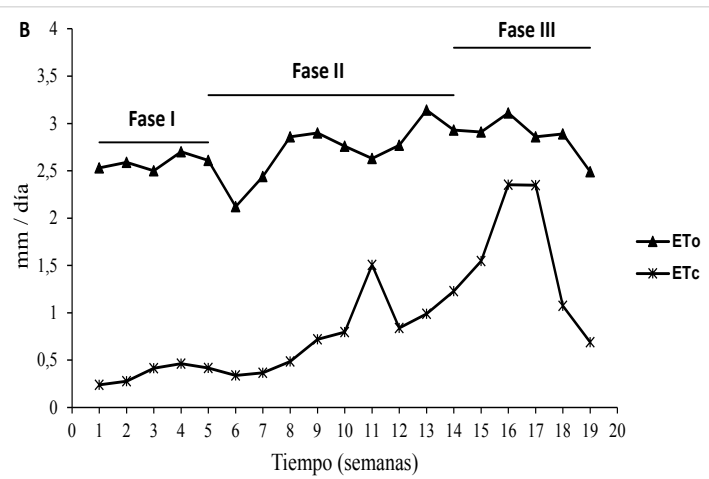

Figura 2. Coeficiente de cultivo para el cultivo de zanahoria. A. Kc en las fases fenológicas. B. ETo y ETc en las fases fenológicas.

La presente investigación registró valores de consumo hídrico total de $106.76 \mathrm{~mm}$ de agua para todo el ciclo. En cada fase el consumo varió según el desarrollo y los requerimientos del cultivo. El consumo de agua en la fase de maduración fue el mayor (Fig. 2); corresponde a la fase donde se presentan las mayores acumulaciones de biomasa y los órganos llegan al final del ciclo. Los datos presentan una tendencia similar a los reportados para cebolla de bulbo en el distrito de riego del Alto Chicamocha (9).

\section{Conclusiones}

El coeficiente de cultivo $(\mathrm{Kc})$ de la zanahoria, bajo las condiciones de Ventaquemada, para las fases fenológicas de crecimiento, elongación y maduración fue de $0.14,0.29$ y 0.55, respectivamente. La fase de crecimiento del cultivo tuvo una duración de 31 días; la de elongación, 58 días, y la de maduración, 36 días. Las necesidades hídricas de la zanahoria fueron de $105.88 \mathrm{~mm}$ de agua durante la totalidad del ciclo de cultivo; el mayor consumo de agua se dio en la fase de maduración del órgano cosechable (0.82), por ende, esta fase es la más susceptible al déficit hídrico, y el menor, en la fase de crecimiento; se recomienda no interrumpir la frecuencia de riego en la fase de maduración.

\section{Referencias}

(1) Rubatzky VE, Quros CF, Simon PW. Carrots and related vegetable Unbelliferae. Crop Production Science in Horticulture Series, $\mathrm{n}^{\circ} 10$. CABI Publishing, 1999; UK. 294 pp.

(2) Suojala T. Pre- and Postharvest Development of Carrot Yield and Quality. Agricultural Research Centre of Finland, Helsinky, 2000. 
(3) FAO. Dirección de Estadística. 2013. Disponible en: http://faostat3.fao.org/download/Q/ QC/S.

(4) Agronet. 2014. Disponible en: http://www. agronet.gov.co/agronetweb $1 /$ Estad \% C3\%ADsticas.aspx.

(5) Domínguez A, de Juan JA, Tarjuelo JM, Martínez RS, Martínez-Romero A. Determination of optimal regulated deficit irrigation strategies for maize in a semi-arid environment. Agric. Water Manage.2012; 110: 67-77. DOI: http:// dx.doi.org/10.1016/j.agwat.2012.04.002.

(6) Gaviola J. Manual de producción de zanahoria. Mendoza, Argentina: INTA; 2013; 97-98.

(7) Bleiholder $\mathrm{H}$, Buhr L, Feller H, Hack R, Klose $\mathrm{H}$, Hess R, Stauss $U$, Meier T, Weber E. Compendio para la identificación de los estadios fenológicos de especies mono- y dicotiledóneas cultivadas - Escala BBCH Extendida.2008. Ciba-Geigy AG. Basilea.

(8) FAO. Evapotranspiración del Cultivo: Guías para la determinación de los requerimientos de agua de los cultivos. Manual No. 56 Serie Riego y Drenaje. 2006; FAO Roma, Italia.

(9) Castro H, Cely G, Vásquez S. Criterios técnicos para un manejo eficiente del riego en cebolla de bulbo, distrito de riego Alto Chicamocha. Boyacá,Colombia: Universidad Pedagógica y Tecnológica de Colombia; 2009.

(10) Pinzón R. Manual para el cultivo de hortalizas. Bogotá: Produmedios; 2012.

(11) Vega Rojas T, Méndez Soto C, Rodríguez Montero W. Análisis del crecimiento de cinco híbridos de zanahoria (D. carota L.) mediante la metodología del análisis funcional. Agronomía Costarricense, 2012; 36(2): 29-46.
(12) Strandberg J. Monitoring growth and development of carro ton organic solis in Florida. Proc. Fla. State Hort. Soc.2001, 114: 307-312.

(13) Matute M, Hoyos P. Influencia de la distribución de las líneas de siembra en las mesetas de cultivo sobre la producción y calidad de la zanahoria CV, Tesis de maestría, Escuela Universitaria de Ingeniería Técnica Agrícola, Universidad Politécnica de Madrid, 2008.

(14) Enciso Z. Evaluación de zanahorias sembradas en verano. Investigación Agraria, 2011; 13(2): 75-79.

(15) Nozumu M. Embrapa Hortalizas. Sistema de Producão. Brasilia, BR, 2000. Disponible en: www.cnph.embrapa.br/public/folders/cultivodecenoura.htm.

(16) Allen RG, Pereira LS, Raes D, Smith M. Evapotranspiración del cultivo: Guías para la determinación de los requerimientos de agua de los cultivos. Estudio FAO Riego y Drenaje No. 56. Organización de las Naciones Unidas para la Agricultura y la Alimentación (FAO). 2006; Roma. 298.

(17) Fernández Gómez R., Ávila Alabarces R, López Rodríguez M, Gavilán Zafra $\mathrm{P}$, Oyonarte Gutiérrez N. Manual de riego para agricultores, Módulo I. Fundamentos de riego. Andalucía. (2010). Signatura Ediciones de Andalucía, S.L.

(18) FAO. La calidad en frutas y hortalizas. En: Manual para la preparación y venta de frutas y hortalizas: del campo al mercado. Depósito de Documentos de la FAO. 2006. Disponible en http://www.fao.org//docrep/006.

(19) Benacchio SS. Algunas exigencias agroecológicas en 58 especies de cultivo con potencial de producción en el Trópico Americano. FONAIAP-Centro Nal. de Inv. Agropecuarias. Ministerio de Agricultura y Cría. Maracay, Venezuela. 1982; 202 p. 